\title{
One Million EEL Spectra Acquisition with Aberration-Corrected STEM: 2-D Chemical Investigation of a Statistically Significant Ensemble of Nanocatalysts
}

\author{
Huolin L. Xin, Julia A. Mundy, ${ }_{* * * *}^{*}$ Randi Cabezas, ${ }_{* * *}^{* *}$ Lena Fitting Kourkoutis, ${ }_{* * *}^{* *}$ David A. Muller, ${ }^{* *}{ }^{* * *}$ \\ Vic Liu, Junliang Zhang, ${ }^{* * *}$ Nalini Subramanian, ${ }^{* * *}$ Rohit Makharia, ${ }^{* * *}$ and Frederick T. Wagner ${ }^{* * *}$ \\ * Department of Physics, Cornell University, Ithaca, NY 14853 \\ ** School of Applied and Engineering Physics, Cornell University, Ithaca, NY 14853 \\ *** Electrochemical Energy Research Laboratory, General Motors, Honeoye Falls, NY 14472
}

Aberration-corrected scanning transmission electron microscopes (STEM) can achieve high probing currents while maintaining an Ångstrom-scale probe for analytical mapping. If aided by another correcting module for improved coupling of the inelastic signal into an electron energy loss spectrometer, the collection efficiency on a thin specimen can be well over $90 \%$. This large usable current allows an electron energy loss spectrum (EELS) with identifiable compositional and bonding information to be recorded in just a few tens of milliseconds and a 64x64-pixel spectroscopic map to be acquired in just a few minutes or less. Using an EELS-optimized system, a $100 \mathrm{KeV}$ Nion UltraSTEM, enabled us to collect more than one million EEL spectra providing chemical maps of hundreds of Pt-Co core-shell structured nanocatalysts before and after fuel cell operations. The unprecedented quantity of data allows us to draw statistically significant conclusions about the chemical microstructure and correlations among microscopic degrees of freedom. We show for example, the preferential segregation of a single atomic layer Pt skin on the $\{111\}$ facets of annealed Pt-Co nanoparticles. However, the complexity of the large and spatially-correlated data sets poses challenges in collection, analysis and presentation. We discuss new and borrowed strategies for elegantly condensing information and providing new opportunities to chemically explore an ensemble of heterogeneous nanomaterials at atomic resolution.

Pt-Co nanoparticles are of particular interest in hydrogen fuel cells, as $\mathrm{Pt}_{3} \mathrm{Co}$ is one of the best catalysts known for the oxygen reduction reaction at the cathode [1-2]. However, the rapid degradation of nanocatalysts at the cathode currently prevents them from meeting the DOE targeted life time. To systematically study the impact of the fuel cell environment on the Pt-Co nanocatalysts, we spectroscopically imaged $\sim 80$ particles as received from the commercial vendor (as-received, Fig. 1a), $\sim 100$ as-received particles that underwent accelerated fuel cell operations (voltage-cycled, Fig. 1b and Fig. 2a), $\sim 60$ as-received particles that were high-temperature annealed (annealed, Fig. 1c) and $\sim 50$ annealed particles that were subsequently acid leached (acid-leached, Fig. 1d). Our analysis of the Pt and Co chemical maps reveals the as-received particles have a $0.77 \pm 0.02 \mathrm{~nm}$ thick, relatively uniform Pt-rich shell surrounding the Pt-Co core. The voltage-cycled particles, on the other hand, demonstrate a significant divergence from the relatively homogenous as-received sample. In particular, the particles on average have grown in size and many particles exhibit multiple Pt-Co cores enclosed in a Pt-rich shell (Fig. 2a), indicating that particle coalescence is an important coarsening mechanism. There is also a strong dependence of the Pt-rich shell thickness on the particle size indicating that not only surface loss, but also Co depletion contributes to the loss of catalytic activity (Fig. 2b). The annealed particles show that the Pt-rich shell is removed (Fig. 1c). However, we find the preferential segregation of an atomically thin Pt rich skin on the $\{111\}$ facet (Fig. 1e and Fig. 1f), the first such spectroscopic identification from an ensemble of nanoparticles. When these particles are subjected to an acid treatment, the Pt rich shell of the as-received sample is restored without facet dependence.

Reference

[1] V. R. Stamenkovic et al., Nat Mater 6, 241 (2007).

[2] S. Chen et al., J Phys Chem C 113, 1109 (2009).

[3] Funded inpart by the Energy Materials Center at Cornell, an Energy Frontier Research Center (DOE \#DE-SC0001086) 


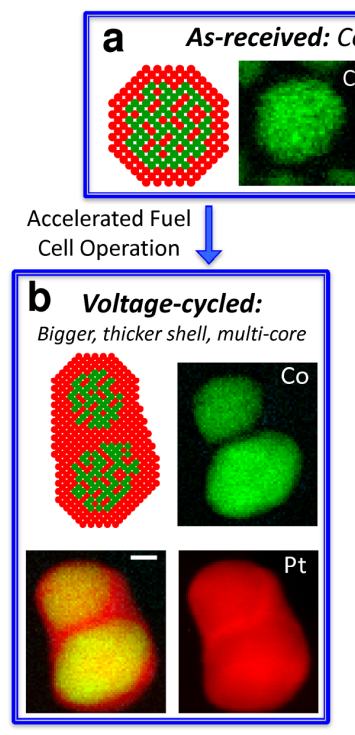

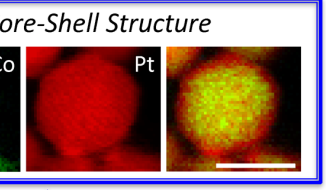
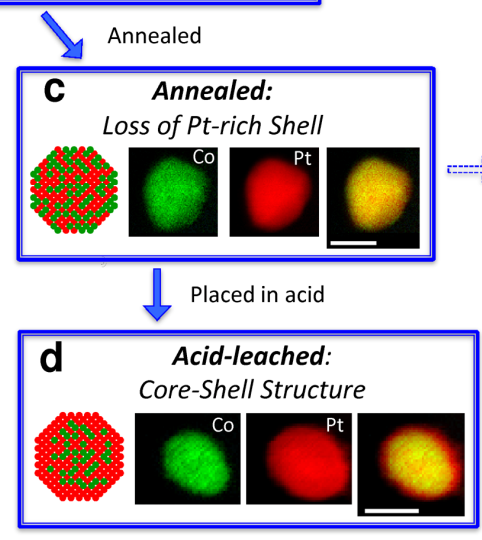
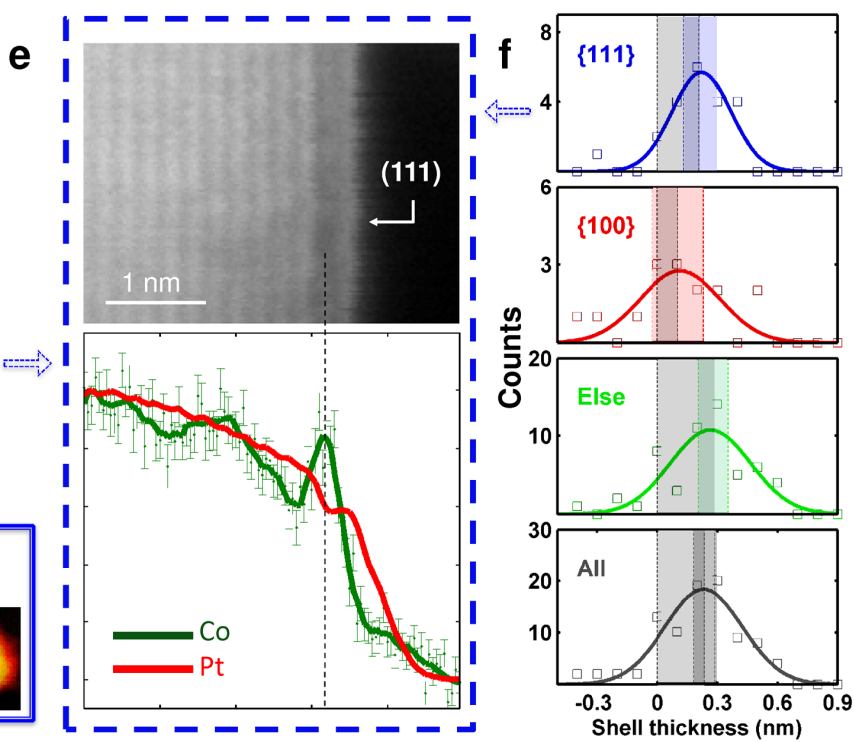

Fig. 1. Chemical mapping and quantification of core-shell structured Pt-Co nanoparticles. (a-d) Pt vs. Co maps of typical particles in the (a) as-received sample, the (b) voltage-cycled sample, the (c) annealed sample and the (d) acid-leached sample (Scale bar: $5 \mathrm{~nm}$ ). In this work, Co concentration map is extracted by subtracting a power law background and integrating over a $\sim 10 \mathrm{eV}$ window around the $\mathrm{L}_{3}$ peak and the Pt map is obtained by multivariate curve resolution on the Pt- $\mathrm{N}_{3}$ edge. (e) A one atomic layer thick Pt-rich skin followed by a Co-rich layer on the (111) facet of an annealed particle is identified spectroscopically with atomic resolution. The EELS line profiles are obtained from a 69x100-pixel spectroscopic image. (f) Facet-dependent analysis of the annealed particles. On the $\{111\}$ surface, the shell thickness is $0.21 \pm 0.04 \mathrm{~nm}$ which is equivalent to one atomic layer thick of Pt in the [111] direction in agreement with the atomic resolution observation in (e). The two ends of the colored bands indicate the $95 \%$ confidence interval.

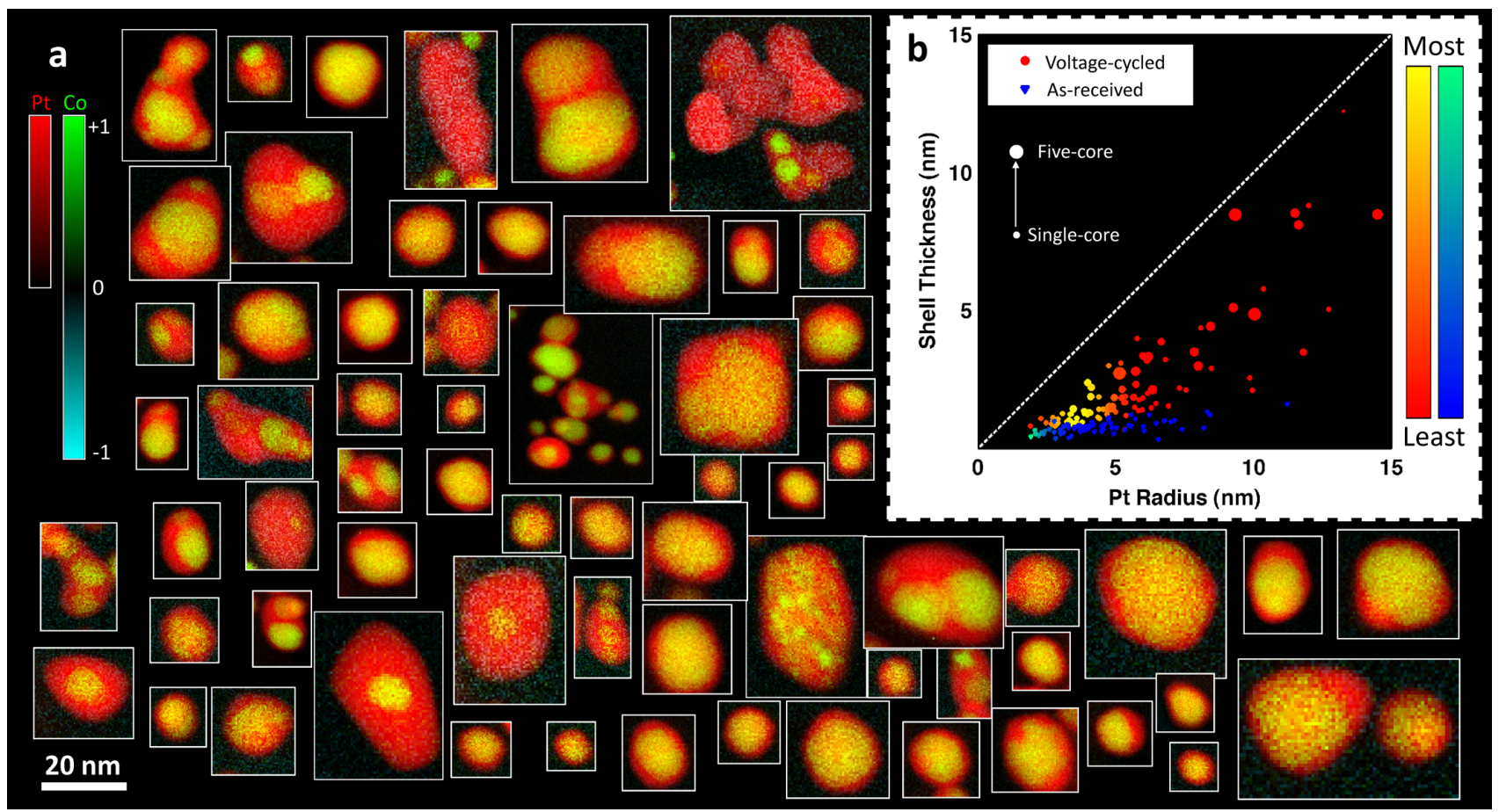

Fig. 2. (a) A collage of the Pt vs. Co maps of the voltage-cycled particles. Only a selected portion is shown here due to space constraint. (b) The quantification of the Pt-rich shell thickness as a function of particle radius of the as-received and voltage-cycled sample. Each dot represents a particle. The dot size indicates the number of distinct Pt-Co cores. The color rendition on the dots represents the particle size distribution (obtained separately) weighted by the surface area. 